\title{
Study of near SOL decay lengths in ASDEX Upgrade under attached and detached divertor conditions
}

\author{
H J. Sun ${ }^{1}$, E. Wolfrum ${ }^{1}$, B. Kurzan ${ }^{1}$, T. Eich ${ }^{1}$, K. Lackner ${ }^{1}$, A. Scarabosio ${ }^{1}$, I. \\ Paradela Pérez $^{1}$, O. Kardaun ${ }^{1}$, M. Faitsch ${ }^{1}$, S. Potzel ${ }^{1}$, U. Stroth ${ }^{1,2}$ and the ASDEX \\ Upgrade Team \\ ${ }^{1}$ Max Planck Institute for Plasma Physics, Boltzmannstr. 2, 85748 Garching, Germany \\ ${ }^{2}$ Physik-Department E28, Technische Universität München, 85747 Garching, Germany \\ E-mail: sunhj@ipp.mpg.de
}

\begin{abstract}
A database with attached, partially detached and completely detached divertors has been constructed of ASDEX Upgrade discharges in both H-mode and L-mode plasmas with Thomson Scattering data suitable for the analysis of the upstream SOL electron profiles. By comparing upstream temperature decay width, $\lambda_{T_{e, u}}$, with the scaling of the SOL power decay width, $\lambda_{q_{\|\|}}$, based on the downstream IR measurements, it is found that a simple relation based on classical electron conduction can relate $\lambda_{T_{e, u}}$ and $\lambda_{q_{\| e}}$ well. The combined dataset can be described by both a single scaling and a separate scaling for H-mode and L-mode. For the single scaling, a strong inverse dependence of, $\lambda_{T_{e, u}}$ on the separatrix temperature, $T_{e, u}$, is found, suggesting the classical parallel Spitzer-Harm conductivity as dominant mechanism controlling the SOL width in both L-mode and $\mathrm{H}$-mode over a large set of plasma parameters. This dependence on $T_{e, u}$ explains why, for the same global plasma parameters, $\lambda_{q_{\|}}$in L-mode is approximately twice that in H-mode and under detached conditions, the SOL upstream electron profile broadens when the density reaches a critical value. Comparing the derived scaling from experimental data with power balance, gives the cross-field thermal diffusivity as $\chi_{\perp} \propto T_{e}^{1 / 2} / n_{e}$, consistent with earlier studies on Compass-D, JET and Alcator C-Mod. However, the possibility of the separate scalings for different regimes cannot be excluded, which gives results similar to those previously reported for the H-mode, but here the wider SOL width for L-mode plasmas is explained simply by the larger premultiplying coefficient. The relative merits of the two scalings in representing the data and their theoretical implications are discussed.
\end{abstract}

PACS numbers: 52.25.Fi, 52.50.Gj

\section{Introduction}

Fusion power plants based on diverted tokamak plasmas must be designed so as to keep the maximum heat flux at the divertor targets within acceptable material limits. Hence, having a reliable predictive basis for this heat flux is an important prerequisite for the design of fusion power plants. It is believed that the divertor heat flux in ITER may constrain operation at highest performance, so a predictive basis is required to ensure optimal ITER operation. The power providing the divertor heat flux 
originates in the core plasma and transfers across the separatrix into the open flux surface region known as the Scrape-off Layer (SOL). In the SOL, the heat flows poloidally, from upstream to the divertor target, as well as radially [1] [2]. In addition to this heat flow, some of the power entering the divertor upstream is dissipated through radiation and charge exchange losses. If the majority of the power entering the divertor is lost through such process leading to a cold diveror plasma $(<5 \mathrm{eV}$ at target) with reduced particle fluxes, the plasma is described as detached. Predicting the heat flux at the divertor target involves developing an understanding of the upstream heat flux and plasma profiles and the transport and loss processes which take place before it reaches the downstream divertor target.

A series of studies of the power flux in the divertor target regions for attached plasmas have been made using Infrared (IR) camera measurements. These began with high confinement mode (H-mode) plasmas in JET, DIII-D, ASDEX Upgrade, C-Mod, NSTX and MAST [3] [4] [5] [6] [7] [8] and have since been extended to low confinement mode (L-mode) plasmas from the two tokamaks JET and ASDEX Upgrade [9] [10]. Regression analyses based on the resulting multi-machine databases for the upstream SOL power fall-off length, $\lambda_{q_{\| e}}$, have shown similar parametric dependencies in $\mathrm{H}$ and Lmode with the most important scaling parameter being the poloidal magnetic field (or equivalently the plasma current in each machine). Interestingly, for the same global plasma parameters, $\lambda_{q_{\| e}}$ in L-mode is approximately twice that in $\mathrm{H}$-mode.

When the plasma density is very low, there is no significant temperature drop along the SOL, and the SOL is said to be in the sheath-limited regime. In this regime, the sheath is the only important element in the edge influencing the transport of particles and power from the confined plasma to the solid surfaces. When ionization of the recycling neutrals occurs in the main plasma, the resulting outflow of plasma gives significant parallel plasma flow, hence parallel heat convection dominates. As the plasma density increases, conduction-limited regime is attained. In this regime, ionization occurs mainly in the SOL and it will also generally occur mainly close to the source of the recycling neutrals, i.e. to the targets. Thus, the role of heat convection is reduced; the parallel conduction carries most of the power flow along the SOL. A simple analytic divertor model - the 'two-point' model with dominant classical conduction is widely used as a useful approach to understand the SOL physics in the conductionlimited regime. Classical heat conduction relates $\lambda_{q_{\| e}}$ and the temperature decay width, $\lambda_{T_{e, u}}$ through the well know results $\lambda_{q_{\| e}}=\frac{2}{7} \lambda_{T_{e, u}}$. Previous study of H-mode in attached condition showed that this relation is consistent with the upstream Te and target IR data indicating a small broadening (not detectable within experimental errors) of this part of the SOL by radial transport [12]. The electron temperature, $T_{e}$, profiles in the near SOL have been found to have a radial exponential decay distribution which, moreover does not vary poloidally. This has also been validated by comparing measurements from the Thomson Scattering (TS) system and IR camera for the same discharges in Lmode plasma [11].

Due to the instrumental constraints, the measurements based on IR thermography used to establish the scalings come from attached divertor discharges over a limited range of operating parameters compared to conditions expected on ITER at high performance. Direct measurements of the upstream decay lengths can provide important complementary information, particularly in detached and partially detached discharges. Compared with the previous H-mode plasma study [12], this paper extends the results to L-mode regimes, focusing on the comparison between the $\mathrm{H}$ - and L-mode regimes and underlying physics. The rest of this paper is organized as follows: The experimental methods are introduced in section 2 and the range of the database is introduced in section 3 . In section 4, the twopoint model is tested against experimental results from TS system and IR camera, and then a single 
scaling for both H-mode and L-mode attached plasmas is derived. In section 5, plasmas under detached divertor conditions are studied. In section 6, the results are summarized and physics implications are discussed.

\section{Experimental method}

In ASDEX Upgrade, high-resolution electron temperature and density profiles can be obtained simultaneously by a vertical Thomson Scattering system [12] [13]. The system is equipped with $4 \mathrm{Nd:}$ YAG lasers at $1064 \mathrm{~nm}$ for core profiles and 6 lasers for the edge plasma. For the classification of divertor detachment, flush mounted Langmuir triple probes (LP) measure the ion saturation current density, $j_{s a t}$, and the electron density, $n_{e, t}$, and the electron temperature, $T_{e, t}$, at the divertor targets. Following reference [14], we define partial detachment as a state of low $T_{e, t}(<5 \mathrm{eV})$ and reduced $j_{s a t}$ at the strike point, and complete detachment as a state with vanishing $n_{e, t}$ at the outer strike point. Further details of these diagnostics and methods are given in reference [14].

In the analysis of TS measurements, data are accumulated over steady state plasma phases of duration 0.5-1.0 s where the global plasma parameters were constant. In H-mode plasma, during ELMs the thermocurrents measured in the divertor are strongly increased, which indicates the onset of an ELM. Before the ELM onset $1.5 \mathrm{~ms}$ are excluded as margin for possible errors in the ELM recognition and time delays between different diagnostics. The relevant period for profile synchronization is defined from -3.5 to $-1.5 \mathrm{~ms}$ relative to the ELM onset time and also at least $4 \mathrm{~ms}$ after the previous ELM are excluded. However, the same method is not applicable for the inter-ELM profiles under the detached condition because the ELM frequency is too high.

Profiles in the SOL region in many devices are often found to exhibit a two-zone structure: a steep gradient region in density and temperature near the separatrix (near SOL) and a flatter profile region (far SOL). In this paper, the study focuses on the features in the steep gradient region, i.e. the near SOL region. In previous studies of the SOL power decay length, based on downstream IR measurements, a purely exponential radial decay of the parallel energy transport was assumed at the divertor entrance. Later studies, based on TS system in attached H-mode plasma on ASDEX Upgrade, found that an exponential profile for the upstream $\lambda_{T_{e, u}}$ in the near SOL is consistent with all the analyzed discharges from H-mode plasmas on ASDEX Upgrade [12]. Experimental results show that this exponential distribution remains in near SOL region of L-mode plasma on ASDEX Upgrade. Based on this fact, the upstream $T_{e}$ decay length $\lambda_{T_{e, u}}$ can be evaluated by a log-linear fit in the near SOL region, as illustrated in Figure 1(a) and Figure 1(b). The resolution of the equilibrium reconstruction is insufficient to determine the position of the separatrix to the accuracy required to determine the electron temperature at the separatrix. Instead, following [12] [15] [16] [17], a power balance has been performed which finds that, across the analyzed dataset in this paper, the electron temperature at the separatrix is around $100 \pm 30 \mathrm{eV}$ for $\mathrm{H}$-mode discharges and around $50 \pm 15 \mathrm{eV}$ for Lmode discharges. Based on this, in common with similar previous studies, in this analysis the separatrix position will be estimated by the point where $T_{e}=100 \mathrm{eV}$ for $\mathrm{H}$-mode and $T_{e}=50 \mathrm{eV}$ for L-mode. As noted in [18] [16], the exponential nature of the electron temperature profile in the near SOL means that the measured decay length does not vary across the near SOL and so the analysis is relatively insensitive to the chosen electron temperature at the separatrix. 

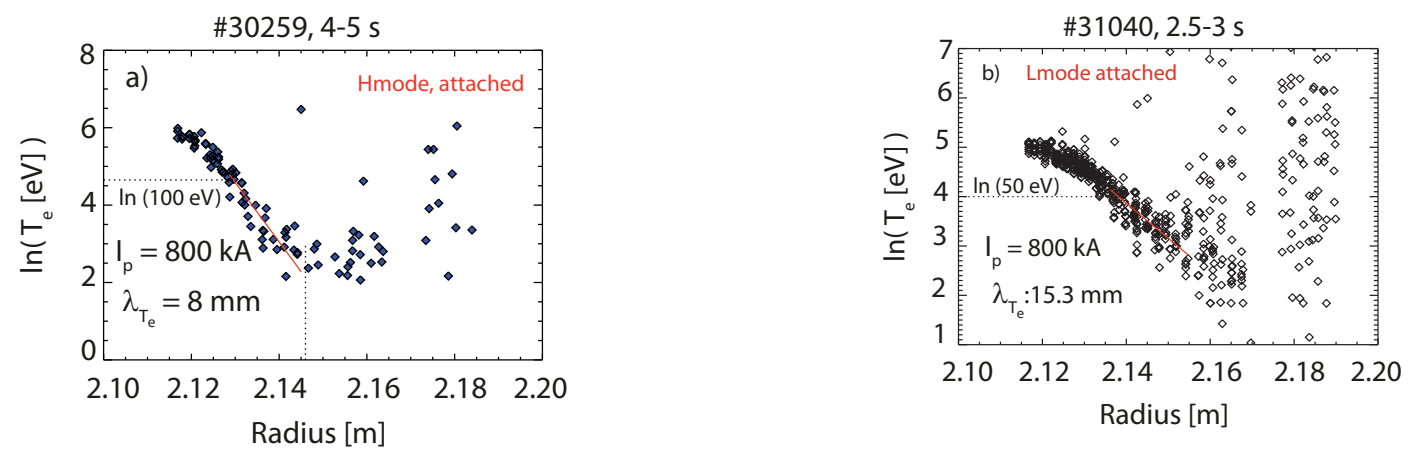

Figure 1. Log-linear plot of the electron temperature against major radius on the midplane for a typical H-mode discharge \#30259 (a), and L-mode discharge \#31040 (b), with fitted line (solid lines) in near SOL. The slope of the fitted line gives the decay lengths, $8 \mathrm{~mm}$ for $\mathrm{H}$-mode and $15.3 \mathrm{~mm}$ for L-mode in same plasma current.

\section{Discharge database}

A database of discharges from the 2014 ASDEX Upgrade campaign was constructed to study upstream near SOL gradient lengths in $\mathrm{H}$-mode and L-mode plasmas. The attached $\mathrm{H}$-mode discharges (36 in total) used in the present dataset are the same discharges as those used in Ref [12]. However, during the preparation of this paper the time windows over which the TS data was taken and the selection of the time points within the time windows were reviewed and, in some cases, revised to improve the quality of the data. This was to address three concerns: firstly, the time windows for some of the discharges were moved to avoid phases of the discharge where plasma parameters, such as density or radiated power, were in any way transient; secondly, it was found that some discharges were affected by inter-ELM fluctuations and time points affected in this way were removed from the analysis; thirdly, some of the time windows were extended to increase the number of data points analysed. In total, 9 of the 36 discharges were revised. The revised calculated $\lambda_{T_{e, u}}$ for the 9 discharges is certainly different, but for most discharges the change is within measurement uncertainties. As a result, the trends and main conclusions of the first paper [12] are still supported by the revised dataset. The revised attached H-mode dataset is used throughout the present paper. In the previous paper, the total heating power $P_{h}$ was used for both the comparison with the $\lambda_{q_{\| e}}$ scaling and the log-linear regression. In this paper, the power entering the SOL, $P_{\text {sol }}$, defined as the total heating power excluding the radiated power in the main plasma, is used in the entire study.

The range of key parameters for the dataset used in this paper is given in table 1 . In the table, $I_{p}$ is the plasma current, $B_{T}$ the toroidal magnetic field, $q_{95}$ the edge safety factor and $P_{s o l}$ is defined above. Since this paper only focuses on ASDEX Upgrade experimental results, the geometric radius of the plasma, $\mathrm{R}_{\text {geo }}$, which is included in the IR-based $\lambda_{q_{\| e}}$ scalings, does not vary greatly within the database and so size scaling is not considered in this paper. It should be kept in mind that this dataset contains only a limited range in $B_{T}$. Although $B_{T}$ varies from 1.9 to $2.56 \mathrm{~T}$; the majority of the dataset for both $\mathrm{L}$-mode and H-mode is comprised of discharges around $2.5 \mathrm{~T}$. This is reflected in the high median value of $B_{T}$ and the fact that the $10 \%-90 \%$ percentiles are heavily skewed towards the top end of the range in both L-mode and $\mathrm{H}$-mode. The poor condition of the dataset with regards to $B_{T}$ results in larger uncertainties in dependencies on $B_{T}$, similar to previous studies IR camera-based $\lambda_{q_{\| e}}$ scaling studies [3]. The range of analyzed values for the dataset used in this paper is given in table 2 . The 
study of plasmas in detached and partially detached divertor conditions reported in Section 5, was performed with a series of discharges with fixed $I_{p}$ (1MA for H-mode and $800 \mathrm{kA}$ for L-mode) and $B_{T}$ $\left(2.5 \mathrm{~T}\right.$ ), with the Greenwald density fraction $f_{G W}$ varying from 0.2 (attached) to 0.6 (completely detached) in L-mode plasma and from 0.45 (attached) to 0.85 (completely detached) in H-mode plasma.

Table 1. Range of key parameters in the database of analyzed discharges, 36 discharges in H-mode attached plasmas and 27 discharges in L-mode attached plasmas. $\mathrm{Q}_{10}-\mathrm{Q}_{90}$ is the spread of the middle $80 \%$ of the data values.

\begin{tabular}{l|ll|ll}
\hline & \multicolumn{2}{|l|}{ H-mode attached discharges } & \multicolumn{2}{l}{ L-mode attached discharges } \\
& range & Median $\left(\mathrm{Q}_{10}-\mathrm{Q}_{90}\right)$ & range & Median $\left(\mathrm{Q}_{10}-\mathrm{Q}_{90}\right)$ \\
\hline $\mathbf{I}_{\mathbf{p}}$ (MA) & $0.6-1.2$ & $0.8(0.6-1.2)$ & $0.6-1.0$ & $0.8(0.6-1.0)$ \\
$\mathbf{B}_{\mathbf{t}}(\mathbf{T})$ & $1.9-2.56$ & $2.5(2.2-2.53)$ & $1.8-2.5$ & $2.47(1.99-2.5)$ \\
$\mathbf{q}_{95}$ & $3.71-7.1$ & $4.73(3.86-6.9)$ & $3.66-6.9$ & $4.87(3.71-6.35)$ \\
$\mathbf{P}_{\text {SOL }}(\mathbf{M W})$ & $1.3-7$ & $2.5(1.49-5.9)$ & $0.3-1.25$ & $0.64(0.35-1.08)$ \\
\hline
\end{tabular}

Table 2. Range of analyzed values in the database

\begin{tabular}{l|lc|ll}
\hline & \multicolumn{2}{|l}{ H-mode attached discharges } & \multicolumn{2}{l}{ L-mode attached discharges } \\
& range & Median $\left(\mathrm{Q}_{10}-\mathrm{Q}_{90}\right)$ & range & Median $\left(\mathrm{Q}_{10}-\mathrm{Q}_{90}\right)$ \\
\hline $\boldsymbol{\lambda}_{\boldsymbol{e}, \boldsymbol{u}} \mathbf{( m m )}$ & $4.2-10.2$ & $7.1(6.0-8.7)$ & $9.8-18.3$ & $13.5(10.1-18.2)$ \\
$\boldsymbol{T}_{\boldsymbol{e}, \boldsymbol{u}}(\mathbf{e V})$ & $87-163$ & $105(91-143)$ & $47-70$ & $61(53-67)$ \\
$\boldsymbol{n}_{\boldsymbol{e}, \boldsymbol{u}}\left(\mathbf{( 1 0}^{\mathbf{1 9}} \mathbf{m}^{-\mathbf{3}}\right)$ & $0.95-5.5$ & $2.0(1.25-3.7)$ & $0.3-1.6$ & $0.98(0.4-1.5)$ \\
\hline
\end{tabular}

\section{$4 \quad$ Experimental results under attached divertor conditions}

\subsection{Simple model relates $\lambda_{q_{\| e}}$ and $\lambda_{T_{e}, u}$ in both confinement regimes}

The purpose of this section is to test whether the upstream and downstream measurements for the complete H-mode/L-mode dataset can be described by the relation $\lambda_{T_{e}, u}=\frac{7}{2} * \lambda_{q_{\| e, u}}$. The relationship is derived from application of Spitzer-Harm parallel thermal conductivity in the conduction limited regime. The relation has previously been shown to describe attached H-mode plasmas in ASDEX Upgrade [12]. Before making the comparison, the dependence of $\lambda_{T_{e, u}}$ on some primary parameters is presented. 
A strong dependence on plasma current $I_{p}$ is found in both H-mode and L-mode plasmas, figure 2(a) and figure 2(b). $\lambda_{T_{e, u}}$ decreases when the plasma current increases. In the figures, different colors and symbols represent different plasma currents. For $I_{p}=600 \mathrm{kA}$ in H-mode plasmas, $\lambda_{T_{e, u}}$ varies considerably between discharges ( 6 discharges in total). These 6 discharges may be used to illustrate how $\lambda_{T_{e, u}}$ changes with other parameters apart from $I_{p}$. The two discharges with the largest $\lambda_{T_{e, u}}$ $(\approx 10 \mathrm{~mm})$, have values of magnetic field and power close to the median values for the dataset: $B_{T} \approx 2.5 \mathrm{~T}$ and $P_{\text {sol }} \approx 2.5 \mathrm{MW}$. There are four discharges (solid red points) with smaller $\lambda_{T_{e, u}}$ $\left(\approx 7 \mathrm{~mm}\right.$ ). Among these, two discharges have a smaller $B_{T} \approx 1.9 \mathrm{~T}$, which align well with the trend seen when $\lambda_{T_{e, u}}$ is plotted against $q_{95}$, figure 3(a). The remaining two discharges have higher $P_{s o l} \approx 5.2 \mathrm{MW}$ and do not align well with the trend in $q_{95}$. For $I_{p}=600 \mathrm{kA}$ and $I_{p}=800 \mathrm{KA}$ in Lmode plasmas, $\lambda_{T_{e, u}}$ are also relatively scattered between discharges with the scatter seems mainly due to the different $B_{T}$ or $q_{95}$, as shown in figure $3(\mathrm{~b})$, where the same data are plotted against $q_{95}$. This suggests that there are other parametric dependences in addition to that on plasma current.

A previous empirical study [4] [10], based on downstream IR measurements, found that $\lambda_{q_{\| e}}$ in ASDEX Upgrade discharges could be well described by the following scalings:

$$
\begin{aligned}
& \lambda_{q_{\| e}}=(0.78 \pm 0.69) B_{T}^{-0.63 \pm 1.05} q_{\mathrm{cyl}}^{1.14 \pm 0.81} P_{\text {sol }}^{-0.05 \pm 0.31} \text { for H-mode attached plasma } \\
& \text { and } \quad \lambda_{q_{\| e}}=(1.45 \pm 0.13) B_{T}^{-0.78} q_{\mathrm{cyl}}^{1.07 \pm 0.07} P_{\text {sol }}^{-0.14 \pm 0.05} \text { for L-mode attached plasma }
\end{aligned}
$$

Here, $\lambda_{q_{\| e}}$ is measured in $\mathrm{mm} ; B_{T}$ is measured in Tesla; $\mathrm{q}_{\mathrm{cyl}}$ is the cylindrical safety factor; and $P_{\text {sol }}$ is measured in MW. In L-mode study [10], since all discharges were conducted at the same $B_{T}$, the dependence of $\lambda_{q_{\| e}}$ on $B_{T}$ was assumed same as in H-mode scaling based on the resulting multimachine databases [3]. The exponent of $B_{T}$ in L-mode scaling is taken directly from the H-mode scaling.

Since IR measurement can only operate in a limited condition, it is very rare that both IR camera and TS system measure same discharges, except some discharges in the L-mode plasma, as presented in Ref [11]. To test the consistency between the IR measurements and the TS measurements, the above empirical scalings can be compared with measured results. In figure 4, it can be seen that, given the uncertainties between different diagnostics, the results are consistent with the two-point model in the conduction limited regime: $\lambda_{T_{e}, u}=\frac{7}{2} * \lambda_{q_{\| e}}$, relates the upstream gradient length to the decay width of the power flux entering the diverted region in conduction-limited regime. Even majority of the database are consistent, some points are offlined, especially the four discharges mentioned earlier which have different $B_{T}$ and $P_{\text {sol }}$, this may imply that the scaling from upstream measurements may have different exponent on these two parameters, comparing with the one based on IR measurements. 

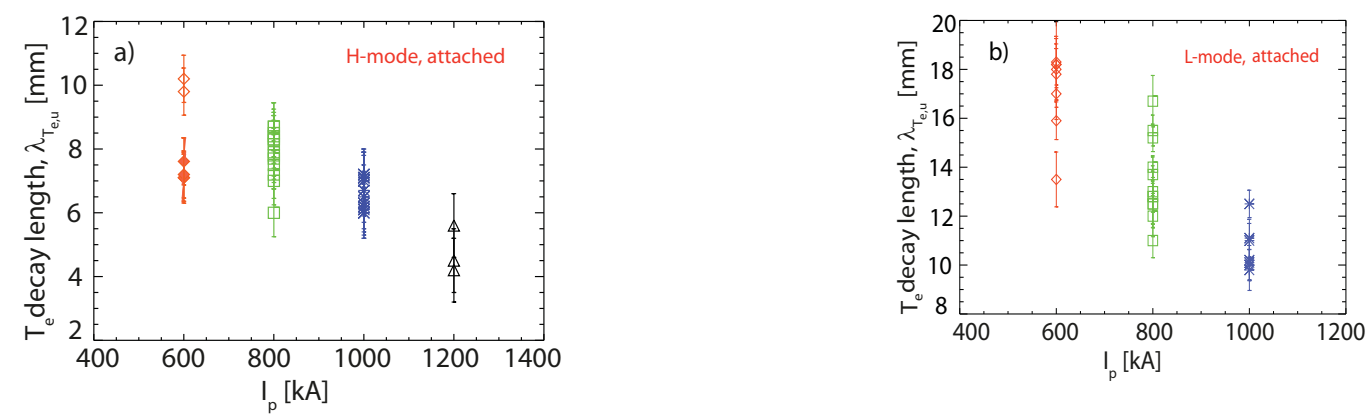

Figure 2. $\lambda_{T_{e, u}}$, against $I_{p}$ : (a) in H-mode attached plasma; (b) in L-mode attached plasma. Different colors and symbols indicate different plasma currents.
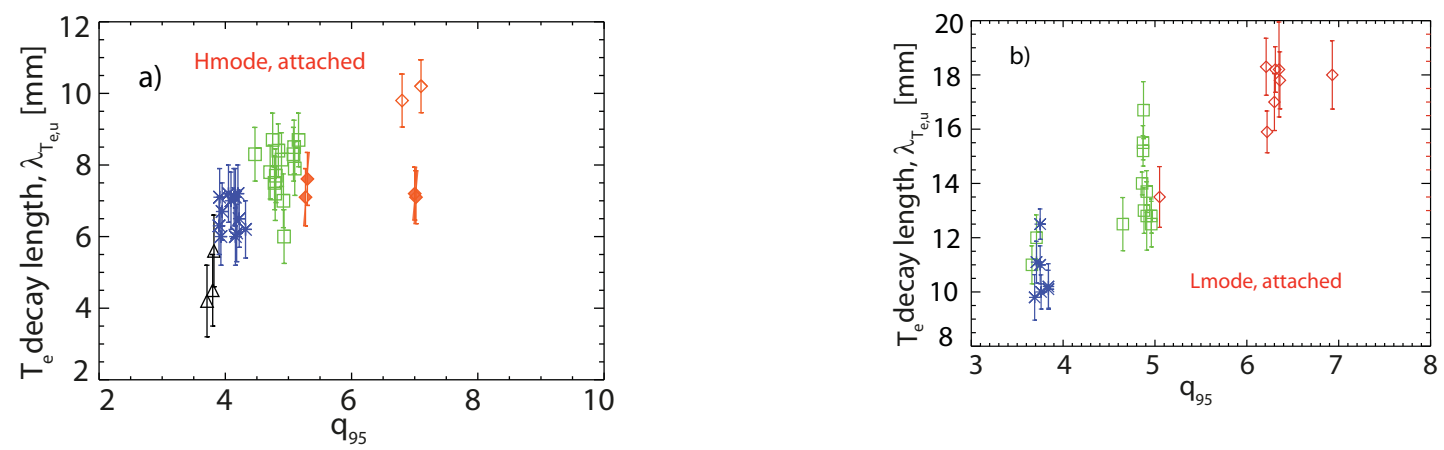

Figure 3. $\lambda_{T_{e, u}}$ against $q_{95}$ : (a) in H-mode attached plasma; (b) in L-mode attached plasma. Different colors and symbols indicate different plasma currents, as illustrated in figure 2.
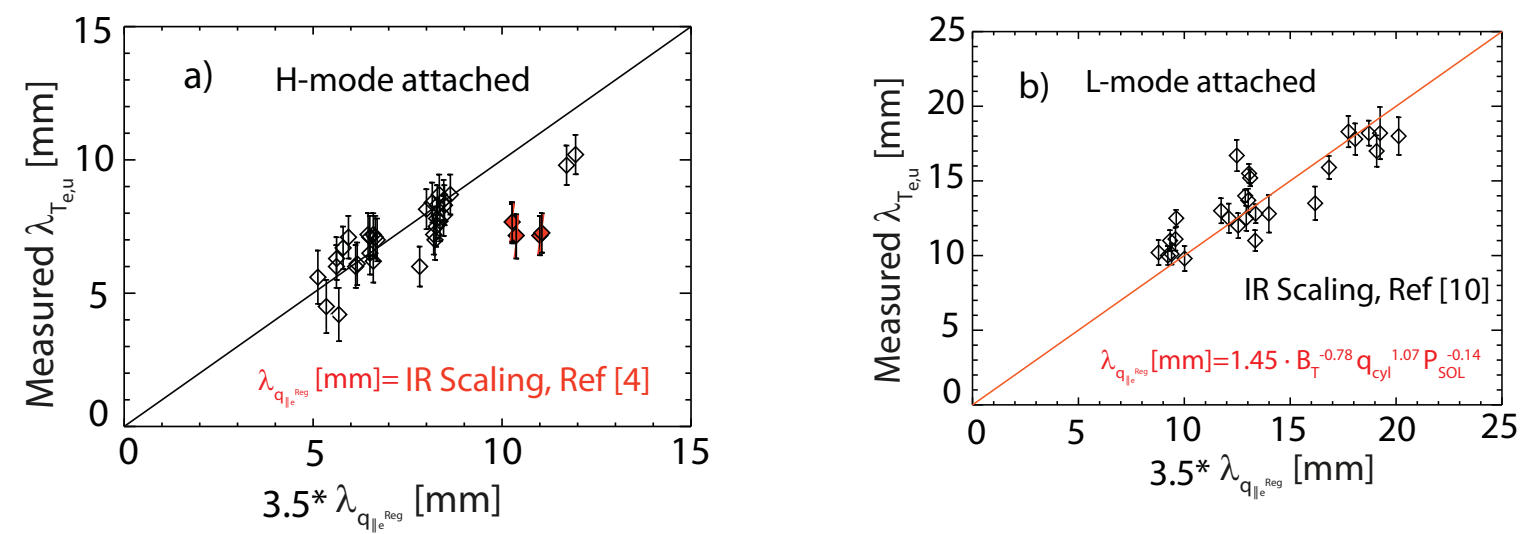

Figure 4. $\lambda_{T_{e, u}}$ from TS measurements against (a) $3.5 * \lambda_{q_{\| e, r e g}}$ scaling from downstream IR measurement for Hmode attached plasma; (b) $3.5^{*} \lambda_{q_{\|, \text {re }} \text { g }}$ scaling from IR measurements for L-mode attached plasma. 


\subsection{A single scaling or separate scalings for $\mathbf{H}$-mode and $\mathbf{L}$-mode regimes?}

In the previous study, the SOL power fall-off lengths based on downstream IR camera have shown similar parametric dependencies in H and L-mode [4] [10]. The first experimental observation from TS system show that the dependences of $\lambda_{T_{e, u}}$ on primary parameters is also similar in H and L-mode regimes. In this section, the question of whether there is correlation between $\mathrm{H}$ and L-mode regimes, apart from similar parametric dependences, will be investigated. Based on the first observation, it will be assumed that $\lambda_{T_{e, u}}$ have the same parametric dependencies in both $\mathrm{H}$ and L-mode regimes. Following previous analyses, log-linear regressions will be made using the form $\lambda_{T_{e, u}}=C \cdot B_{T}^{C_{B}} \cdot q_{95}^{C_{q}}$. $P_{s o l}^{C_{P}}$. To study the degree to which H-mode and L-mode regimes differ, two different approaches will be taken: firstly, assuming a single scaling works for both $\mathrm{H}$-mode and L-mode regimes, i.e. $C_{H}=C_{L}$; secondly, assuming different scalings between these two regimes, i.e. $C_{H} \neq C_{L}$.

\subsubsection{One single scaling for both $\mathrm{H}$ and $\mathrm{L}$-mode plasma, $C_{H}=C_{L}$}

Performing a fit to the combined H-mode and L-mode attached plasma dataset gives the scaling:

$$
\lambda_{T_{e, u}}=3.32 \cdot q_{95}^{0.64 \pm 0.13} P_{\text {sol }}^{-0.32 \pm 0.05} B_{T}^{0.23 \pm 0.35} .
$$

The regression has a fit quality of $\mathrm{R}^{2}=0.87$ (RMSE: 13\%). As shown in figure 5 (a), the dataset is well represented by the scaling with no obvious systematic deviations. Due to the relatively small variation in IR based ASDEX Upgrade datasets, the parametric dependencies have large uncertainties with $B_{T}$ and $P_{\text {sol }}[4]$ [10]. The dataset combining both H-mode and L-mode regimes, considerably extends the range of $P_{s o l}$, resulting in a dependence with much smaller uncertainty. However, it should be emphasized that the $\mathrm{B}_{\mathrm{T}}$ dependence still has a large uncertainty due to the small variation in $\mathrm{B}_{\mathrm{T}}$, as for the previous IR and TS based ASDEX Upgrade datasets. Excluding $\mathrm{B}_{\mathrm{T}}$ gives:

$$
\lambda_{T_{e, u}}=3.94 * q_{95}^{0.66 \pm 0.13} P_{\text {sol }}^{-0.3 \pm 0.027} .
$$

This has the same fit quality of $\mathrm{R}^{2}=0.87$ (RMSE: $13 \%$ ) as the three parameter regression, as shown in figure 5(b). This may indicate that the present database can not give a reliable $B_{T}$ dependence. Thus, the following work will examine the differences in the estimates of the $P_{\text {sol }}$ dependence, while the variation of the dependence on $B_{T}$ will not be discussed in this paper.

The scaling for the combined H-mode and L-mode attached plasma dataset differs from the previous single regime scalings [4] [10] [12]in having an, albeit relatively weak, dependence of $\lambda_{T_{e, u}}$ on $\mathrm{P}_{\mathrm{SOL}}$, for fixed field and plasma current. In previous scaling in H-mode attached plasma [12], the total heating power $P_{\text {heat }}$ was used instead of $P_{\text {sol }}$. They are correlated but they have different trend within. Regarding the difference between the dependences on $q_{95}$ and $B_{t}, \mathrm{~B}_{\mathrm{T}}^{-0.5 \pm 0.67} \mathrm{q}_{95}^{0.97 \pm 0.17}$ and $\mathrm{B}_{\mathrm{T}}^{0.23 \pm 0.35} \mathrm{q}_{95}^{0.64 \pm 0.13}$ are consistent because $q_{95}$ is correlated with $B_{t}, q_{95} \sim B_{T}^{1.0}$. Interestingly, the $P_{\text {sol }}$ dependence of $\lambda_{T_{e, u}} \propto P_{\text {sol }}^{-0.32 \pm 0.05}$ is very similar to a recent statistical analysis of a significantly enlarged database in limiter configuration (eleven tokamaks) [19]. Regression analysis of this same database with dedicated measurements of $\lambda_{q_{\| e}}$ extracted from Langmuir probe show that the best engineering scaling has a dependence of $\lambda_{q_{\| e}} \propto P_{\text {tot }}^{-0.38}[19]$. 

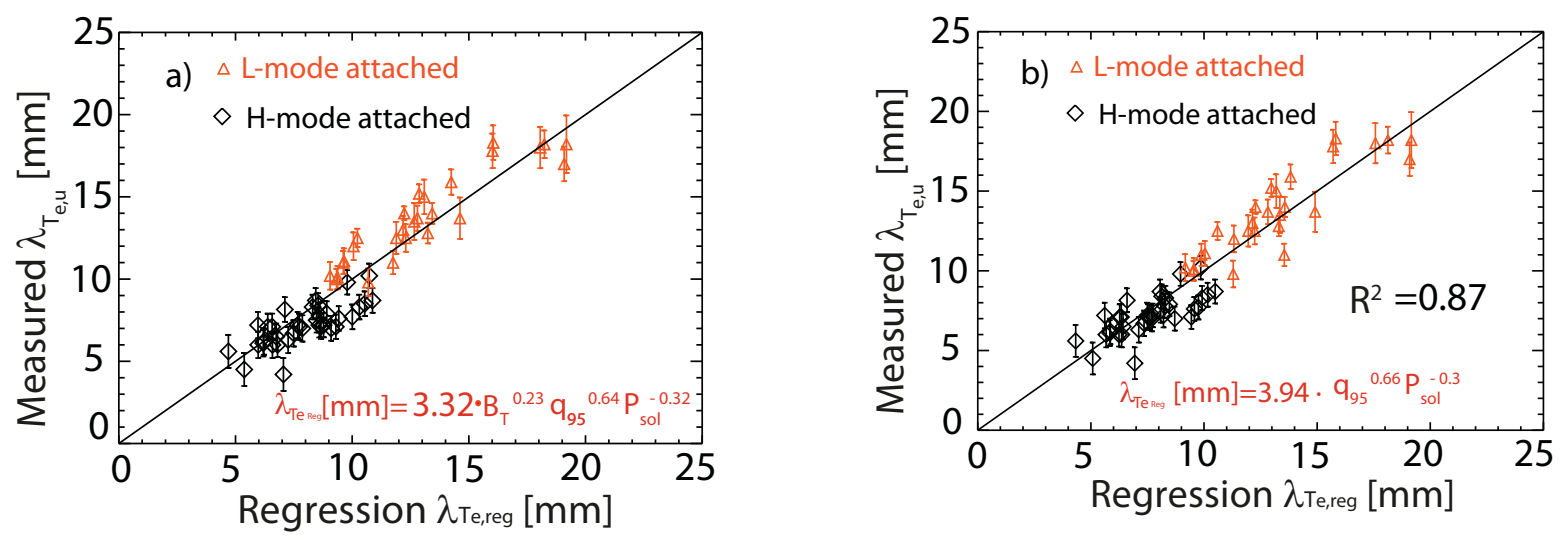

Figure 5. Measured $\lambda_{T_{e, u}}$ against a single scaling for both $\mathrm{H}$ and L-mode regimes, with (a) three parameters: $\lambda_{T_{e, u}}=3.32 * q_{95}^{0.64 \pm 0.13} P_{s o l}^{-0.32 \pm 0.05} B_{T}^{0.23 \pm 0.35}$ (b) two paramters: $\lambda_{T_{e, u}}=3.94 * q_{95}^{0.66 \pm 0.13} P_{\text {sol }}^{-0.3 \pm 0.05}$

As shown in Section 4.1, a dominant Spitzer-Harm conduction, $q_{\|}=-\kappa_{0} e^{T_{e}}{ }^{\frac{5}{2}} \nabla_{\|} T_{e}$, can explain the relation between $\lambda_{q_{\| e}}$ and $\lambda_{T_{e, u}}$. Given its prominence and strong dependence on $T_{e, u}$, it should be expected that $T_{e, u}$ plays also a role in determining the SOL width itself (since the SOL width is believed to result from the competition of parallel and perpendicular transport). To test this assumption, SOLPS simulations [20] of ASDEX Upgrade H-mode like plasma with spatially varying but otherwise parameters-independent perpendicular transport coefficients show the expected relationship between $\lambda_{T_{e}, u}$ and $T_{e, u}$ (see figure 6). $T_{e, u}$ is varied by scanning the upstream density (gas puff) and $\lambda_{T_{e}, u}$ is found to scale, within uncertainties, as $T_{e, u}^{-1.25}$. The results support the importance of classical parallel electron conduction also in a model (basically the Braginskii equations) including ion and convective transport, as well as neutron physics, indicating that some of the complex physics, although not included in the simple analysis of the SOL but present in SOLPS, have a small influence on the link between upstream SOL width and local plasma parameters. Thus, in the following experimental analysis, classical conduction will be considered as the only parallel heat transport mechanism.

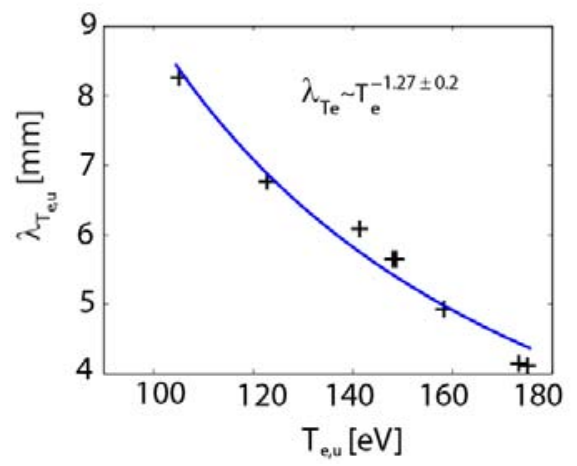

Figure 6. $\lambda_{T_{e, u}}$ against $T_{e, u}$, SOLPS simulation results of ASDEX Upgrade H-mode like plasma. It show a strong inverse correlation between $\lambda_{T_{e}, u}$ and $T_{e, u}$, as expected from Spitzer-Harm conduction. 
Based on the classical Spitzer-Harm conduction, the equation below can be used to estimate the separatrix temperature, $T_{e, u}[2]$ :

$$
T_{e, u} \cong\left(\frac{7}{2} \frac{\left(P_{s o l} / A_{q_{\|}}\right) L}{\kappa_{0 e}}\right)^{2 / 7}
$$

Here, the simplified relation $L=\pi R q_{95}$ is used to calculate the connection length and $A_{q_{\|}}=$ $4 \pi R \lambda_{q} B_{\theta} / B_{T}$ is the surface area for the parallel power flux. $\lambda_{q_{\| e}}=\frac{2}{7} \lambda_{T_{e}, u}$ is used to calculate $A_{q_{\|}}$. Thomson Scattering measures the electron temperature and density simultaneously, and so the calculated $T_{e, u}$, can then be used to determine the position of separatrix and, thus, the upstream density, $n_{e, u}$. Applying equation 3 to the scaling in equation 1 gives $\lambda_{T_{e}, u} \propto q_{95}^{0.95} T_{e, u}^{-0.85}$, which is close to the simple form $\lambda_{T_{e}, u} \propto q_{95} T_{e, u}^{-1}$. Figure 7 (a) $\lambda_{T_{e}, u}$ normalized by $q_{95}$ against $T_{e, u}$ calculated from equation 3. In this figure, the black dotted line describes the relation $\lambda_{T_{e}, u} / q_{95} \propto T_{e, u}^{-0.85}$. (The blue dashed line describes $\lambda_{T_{e}, u} / q_{95} \propto T_{e, u}^{-1 / 2}$, which will be explained in the next section). The parametric dependence of the combined H-mode and L-mode dataset can be well described by a negative dependence in $T_{e, u}$. However, there is no obvious correlation with $n_{e, u}$, as shown in figure 7(b). The negative dependence in $T_{e, u}$ can well explain why, for the same global plasma parameters, the SOL width in L-mode is approximately twice that in H-mode.
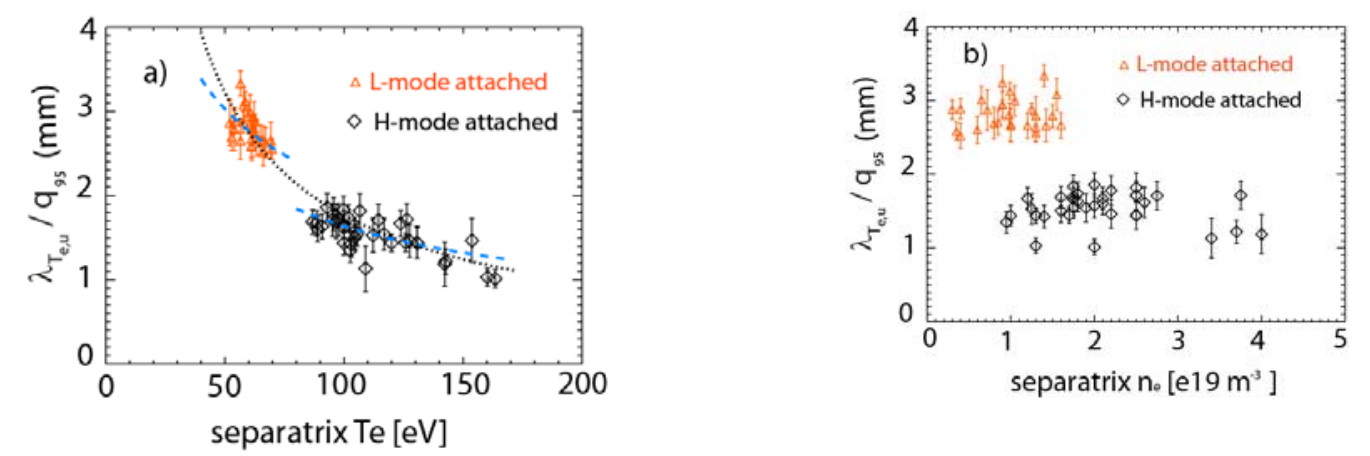

Figure 7. $\lambda_{T_{e, u}}$, normalized by $q_{95}$ against (a) separatrix $T_{e, u}$ and (b) density $n_{e, u}$.

In the SOL, the simultaneous flow of the power poloidally, from the upstream to the divertor target, as well as radially, can be described by:

$$
P_{S O L}=q_{\|} A_{\|}=q_{\perp} A_{\perp} .
$$

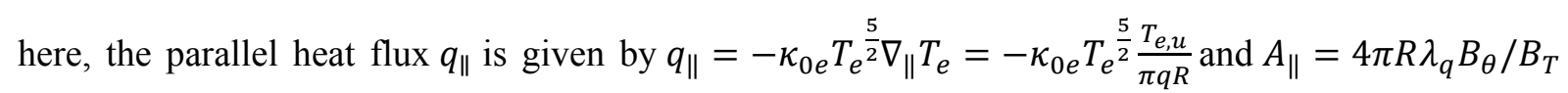
is the surface area for the parallel flux. The cross-field perpendicular heat flux $q_{\perp}$ can be expressed as $q_{\perp}=-K_{\perp} \nabla_{\perp} T_{e}=-K_{\perp} \frac{T_{e, u}}{\lambda_{T_{e, u}}}$, where, $K_{\perp}=n_{e, u} \chi_{\perp}$ is the perpendicular conductivity, $\chi_{\perp}$ is the perpendicular thermal diffusivity, and the surface area for the perpendicular flux is $A_{\perp}=2 \pi a \cdot 2 \pi R$. Equation (4) then gives

$$
\chi_{\perp} \propto n_{e, u}^{-1} \lambda_{T_{e, u}}^{2} q_{95}^{-2} T_{e, u}^{5 / 2} R^{-2} .
$$


Here, the geometric radius of the plasma, $\mathrm{R}$, does not vary greatly within the database, so size dependence will not be considered in this study. Combining equation 5 with the approximate relation implied by the dataset, $\lambda_{T_{e}, u} \propto q_{95} T_{e, u}^{-1}$, gives $\chi_{\perp} \propto T_{e}^{1 / 2} / n_{e}$. This is consistent with earlier studies [21] [22] [23] [24]. Previous results [22] of JET, taken from direct measurements using fast reciprocating probes, give an approximate correlation, $\chi_{\perp}^{S O L} \propto n_{e, u}^{-1}$. The studies [23] comparing theoretical models for the cross-field thermal diffusivities with SOL data from Compass-D, JET and Alcator C-Mod, found that the theories with a $\chi_{\perp} \propto T_{e}^{1 / 2} / n_{e}$ scaling best described the experimental data.

\subsubsection{Different scalings for $\mathrm{H}$ and L-mode plasmas, $C_{H} \neq C_{L}$}

An obvious different slope on natural $\ln$ scale for $\lambda_{T_{e, u}} / q_{95}^{0.64}$ against $P_{s o l}$ is not observed between attached H- and L-mode plasma. So, even assuming different scalings for H- and L-mode plasmas, it is reasonable to assume that $\lambda_{T_{e, u}}$ have same parametric dependencies in both $\mathrm{H}$ and L-mode regimes, only with different coefficients for different regimes. A log-linear regression over the combined $\mathrm{H}$ mode and L-mode dataset gives the following best fit scaling:

$$
\lambda_{T_{e, u}}=C_{H, L} q_{95}^{0.75 \pm 0.12} P_{\text {sol }}^{-0.15 \pm 0.06} B_{T}^{0.11 \pm 0.24}\left(C_{H}=2.4 ; C_{L}=3.53\right)
$$

This regression has a fit quality of $\mathrm{R}^{2}=0.95$ (RMSE: 9\%). As shown in figure 8 , the dataset is also well represented by this scaling with no obvious systematic deviations. Comparing to the scaling in equation 1 , within the uncertainties, the $q_{95}$ and $B_{T}$ dependencies agree with each other, but $P_{\text {sol }}$ dependence is weaker which causes the difference of coefficients in different regimes.

Applying equation 3 to the scaling in equation 6 gives $\lambda_{T_{e}, u} \propto C_{H, L} q_{95}^{0.91} T_{e, u}^{-0.46}$, which is close to $\lambda_{T_{e}, u} \propto C_{H, L} q_{95} T_{e, u}^{-0.5}$. In Figure 7 (a), the blue dashed lines are $\lambda_{T_{e}, u} / q_{95} \propto T_{e, u}^{-0.5}$. It seems like the scaling 6 can also be interpreted by a correlation with $T_{e, u}$ as the scaling 1 , but with a weaker dependence.

Combining equation 5 with the expression $\lambda_{T_{e}, u} \propto C_{H, L} q_{95} T_{e, u}^{-0.5}$, gives $\chi_{\perp} \propto \frac{C_{H, L}^{\chi} T_{e}^{\frac{3}{2}}}{n_{e}}\left(C_{H}^{\chi}\right.$ for H-mode plasma, $C_{L}^{\chi}$ for L-mode plasma). In this case, the cross field transport has same parametric dependences in $\mathrm{H}$ - and L-mode plasma, but with smaller coefficient for $\mathrm{H}$-mode plasma.

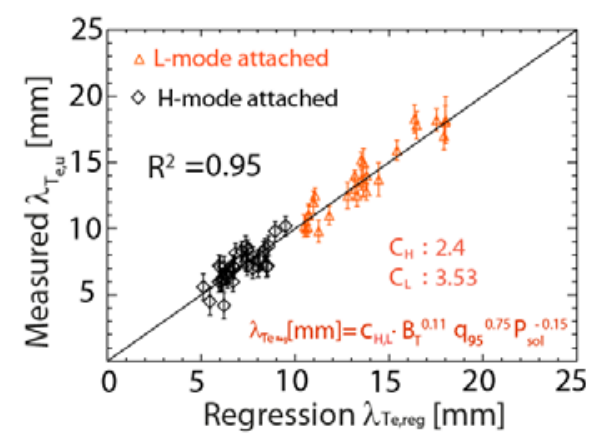

Figure 8. Measured $\lambda_{T_{e, u}}$ against the scaling with different coefficients for $\mathrm{H}$ and L-mode plasma, $\lambda_{T_{e, u}}=$ $C_{H, L} q_{95}^{0.75 \pm 0.12} P_{\text {Sol }}^{-0.15 \pm 0.06} B_{T}^{0.11 \pm 0.4}\left(C_{H}=2.4 ; C_{L}=3.53\right)$ 


\subsubsection{Comparison of the two models}

Determining which type of scaling describes the data is important for understanding the underlying physical mechanism. The main difference between the scalings in equation 1 and 6 is in the $P_{\text {sol }}$ dependence. A single scaling with a stronger dependence on $P_{\text {sol }}^{-0.32}$, fits both $\mathrm{H}$ and L-mode plasmas, while the separate H-mode and L-mode datasets are better described by a weaker power dependence, $P_{s o l}^{-0.15}$, but with different coefficients for the two regimes. However, it can be seen that the difference between the power scalings is small and equivalent to approximately 2 standard deviations in the uncertainties of the fits. The separate scalings for H-mode and L-mode gives a somewhat better fit to the data $\left(\mathrm{R}^{2}=0.95\right)$ than the combined scaling $\left(\mathrm{R}^{2}=0.87\right)$, but this is largely explained by the fact that the additional free parameter in the separate scaling naturally gives rise to a better fit. A direct way to test the validity of the power dependence would be by looking at plasmas with matched field and plasma current that have markedly different $\mathrm{P}_{\mathrm{SOL}}$. For the same current, the range of $P_{\text {sol }}$ is very small, typical 2 times, equivalent to around $20 \%$ variation in $P_{\text {sol }}^{-0.32}$ and $10 \%$ variation in $P_{\text {sol }}^{-0.14}$, which is difficult to observe within diagnostic accuracy. SOL in the H-mode attached regime is much thinner, making it more difficult to detect variation of the decay length associated with the change of $P_{\text {sol }}$. When the safety factor is relatively large, the SOL in L-mode plasma is relatively thick; giving the possibility to observe how the SOL temperature profile varies with $P_{\text {sol }}$. A log-linear plot of the electron temperature profile for a L-mode plasma with $I_{p}=600 \mathrm{kA}, q_{95}=6.3$ and $P_{S O L} \approx 0.3 \mathrm{MW}$ under attached divertor conditions is shown in figure 9(a), with a temperature decay length of $\lambda_{T_{e, u}} \approx$ $18 \mathrm{~mm}$. And in figure $9(\mathrm{~b})$, a discharge with same $I_{p}$, but slightly lower $q_{95}=5$ and higher $P_{S O L} \approx$ $0.55 \mathrm{MW}$, has much thinner SOL, $\lambda_{T_{e, u}} \approx 12.5 \mathrm{~mm}$. The variation among these two discharges is more consistent with the regression result $\lambda_{T_{e}, u} \propto q_{95}^{0.64} P_{S O L}^{-0.32}$, giving support to the single scaling. However, a single example is insufficient to clarify the $P_{\text {sol }}$ dependence, especially as there is some variation in $q_{95}$ and $B_{T}$. Thus, more dedicated experiments scanning $P_{\text {sol }}$ with the same other global parameters is needed for further validation.
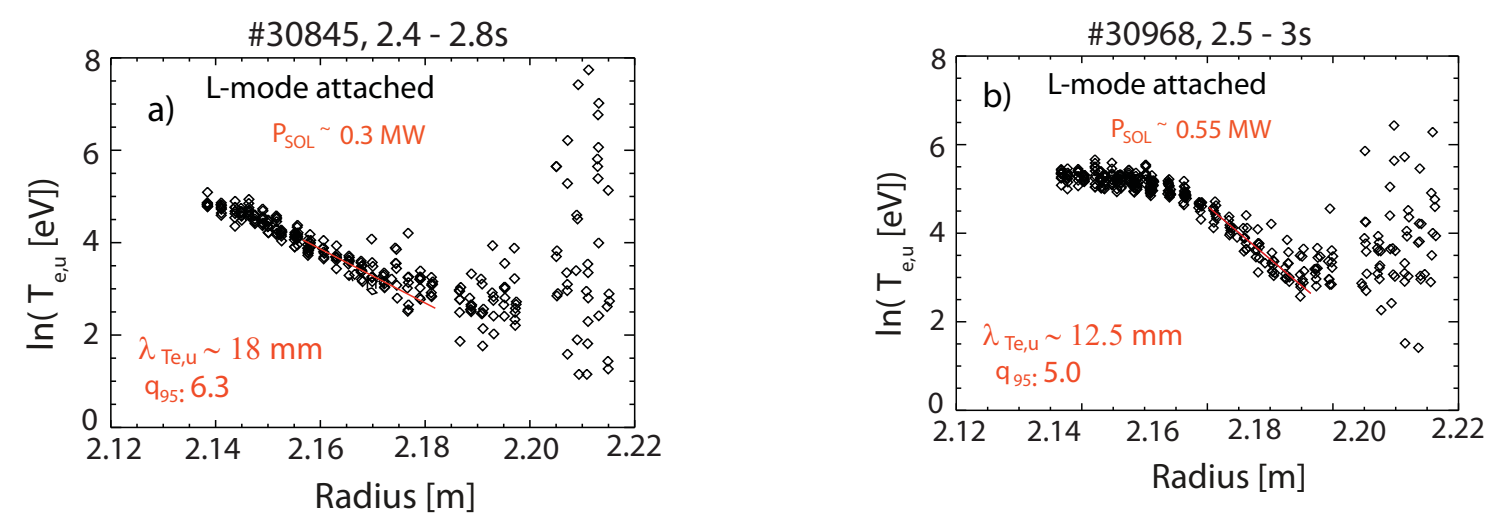

Figure 9. Log-linear plot of the electron temperature against major radius on the mid-plane for L-mode attached discharges with $I_{p} \approx 600 \mathrm{kA}$ : (a) \#30845 with lower $P_{\text {sol }} \approx 0.3 \mathrm{MW}$, and (b) \#30968 with lower $q_{95} \approx 5$ and higher $P_{s o l} \approx 0.55 \mathrm{MW}$. The slope of the near SOL profile gives $\lambda_{T_{e, u}}$. 


\section{Experimental results under detached divertor conditions}

To achieve the detached and partially detached divertor condition, a dedicated density scan with fixed current (800 kA for L-mode, $1 \mathrm{MA}$ for H-mode) and fixed magnetic field (2.5 T) was performed. The onset of partial detachment and complete detachment is discussed in section 2. In the previous study with H-mode plasma [12], the near SOL electron density and temperature profiles are observed to broaden. This only happens when the divertor is completely detached. This phenomenon is similar to the one reported in earlier studies with L-mode plasmas, referred to as the L-mode high density transition (HDT) [25] [26]. This paper only considers the temperature profile in the near SOL and follows the line of the scaling study under attached divertor condition. Thus, the correlation between the broadening of upstream temperature profile and the HDT phenomenon will not be discussed. The observed decay lengths for the density scans are shown in figure 10(a) for H-mode and figure 10(b) for L-mode. For both H- and L-mode plasma, the flattening of the temperature profile does not happen at the onset of partial detachment, but does happen under completely detached conditions. Due to the increase of plasma radiation under detached divertor conditions, the power entering the SOL region starts to decrease. Under completely detached divertor condition, the radiation increases dramatically and, as a result, $P_{\text {sol }}$ decreases significantly. In the H-mode completely detached discharges with very high density, $P_{s o l}$ drops to levels similar to those for L-mode attached plasma. Meanwhile, $\lambda_{T_{e, u}}$ for these completely detached discharges increase to the similar values for L-mode attached discharges, as shown in figure 10(a) and figure 10(b). This suggests that there is correlation between $P_{s o l}$, and $\lambda_{T_{e}, u}$, just as the results for plasmas under attached divertor condition. In Section 4, it has been shown that, under attached divertor conditions, the dependence of $\lambda_{T_{e, u}}$ on $P_{s o l}$ can be described by negative dependence in $T_{e, u}$. To study its impact on detached and partially detached plasmas, it is assumed that the parallel heat transport is still dominated by collisional conduction, hence, equation (3) is still suitable to estimate $T_{e, u}$. Figure 11(a) and figure 11(b) show $\lambda_{T_{e, u}}$ against $T_{e, u}$ for the same discharges shown in figure 10(a) and figure 10(b). Under detached conditions, the discharges with broadened profiles have much lower $T_{e, u}$ due to much lower $P_{s o l}$, while those with unchanged profiles have similar temperature at the separatrix with attached plasma. When combining attached and detached plasmas, the relation $\lambda_{T_{e}, u} / q_{95} \propto T_{e, u}^{-0.85}$ can fit the majority of data, except the L-mode detached regime discharges, as shown in figure 12. This could be due to the change of cross-field transport under detached conditions being significant in L-mode, while remaining relatively small in $\mathrm{H}$-mode. The relation $\lambda_{T_{e}, u} / q_{95} \propto T_{e, u}^{-0.5}$ does not describe well the combined attached and detached plasma datasets. In addition, the expected relation between $\lambda_{T_{e, u}}$ and $T_{e, u}$ is not very clear for attached plasma in figure 11, this could be due to the neglected ion loss channel in $P_{s o l}$, which brings uncertainty in the calculated $T_{e, u}$. 

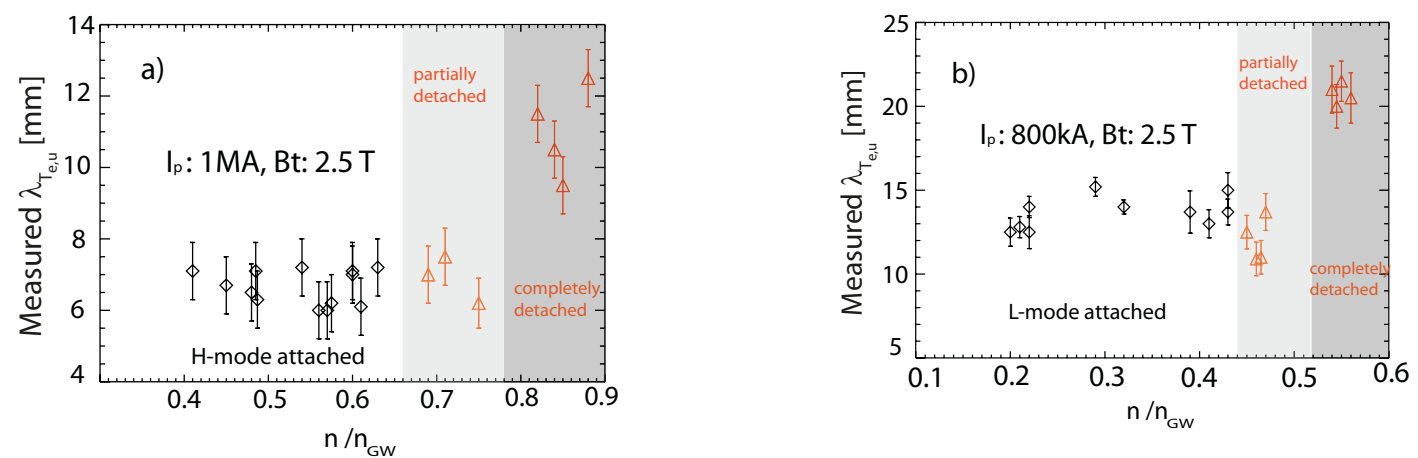

Figure 10. $\lambda_{T_{e, u}}$ against Greenwald density fraction for: (a) H-mode plasmas; and (b) L-mode plasmas. Light and dark gray indicate partially detached and completely detached divertor conditions.
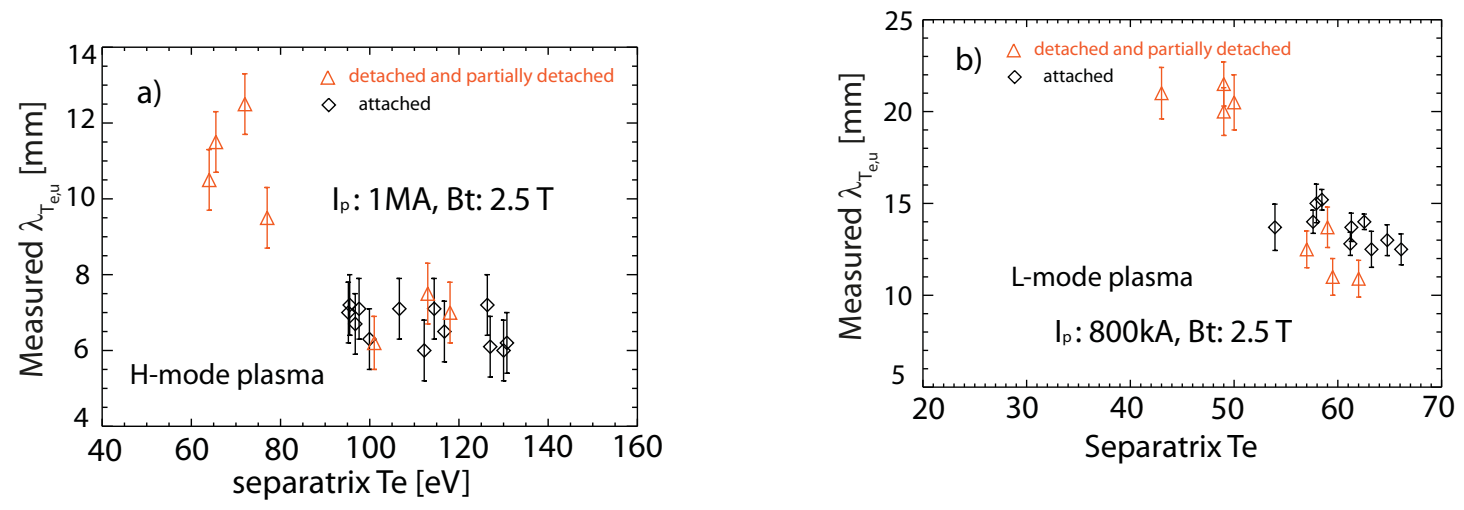

Figure 11. $\lambda_{T_{e, u}}$ against $T_{e, u}$, for: (a) H-mode plasmas; and (b) L-mode plasmas.

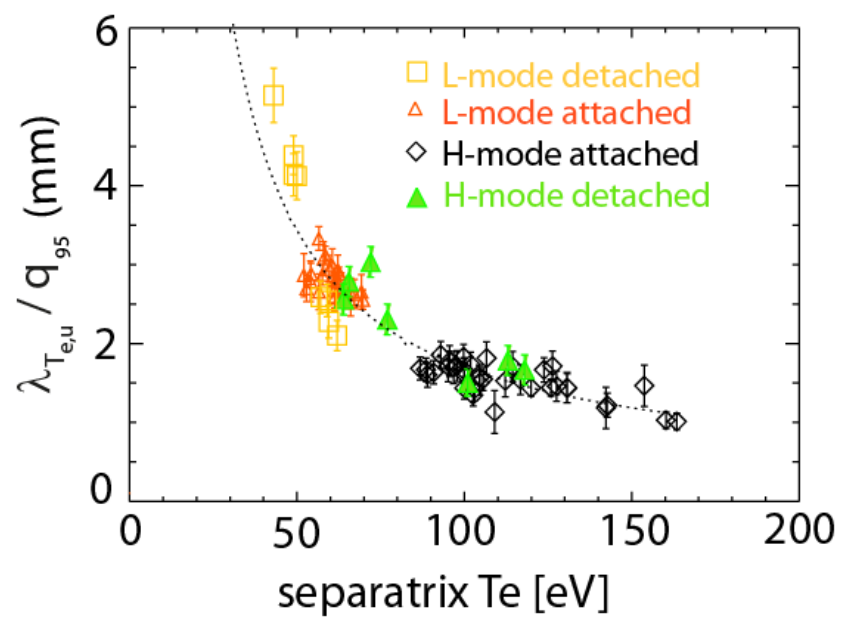

Figure 12. $\lambda_{T_{e, u}}$ normalized by safety factor against $T_{e, u}$.

\section{$6 \quad$ Summary and discussion}

A database of H- and L-mode discharges has been constructed of ASDEX Upgrade with Thomson Scattering data suitable for the analysis of upstream SOL electron profiles. Plasmas with attached, partially detached, and completely detached divertors are included and have been analyzed. By 
comparing upstream, $\lambda_{T_{e, u}}$, with the scaling deduced from downstream IR measurements, it is found that the results are consistent with Spitzer-Harm parallel conduction relating $\lambda_{q_{\| e}}$ and $\lambda_{T_{e, u}}$. This means that the ASDEX Upgrade dataset is in the conduction-limited regime, i.e. the parallel transport in SOL is dominated by Spitzer-Harm conduction. Interestingly, recent study in inboard-limited plasmas in TCV also shows that classical conduction plays essential role in the SOL [27]. Previous studies based on downstream IR camera and the first observation from TS system shows that the SOL decay widths have similar parametric dependences in $\mathrm{H}$ - and L-mode plasmas. Based on this, two different assumptions have been made to study the correlation between H- and L-mode SOL plasmas: assuming one single scaling for both regime, and assuming similar parametric dependences with different coefficient for each regime. A single scaling $\lambda_{T_{e}, u} \propto C_{H L} q_{95}^{0.64} P_{S O L}^{-0.32}\left(C_{H L}\right.$ for both $\mathrm{H}-$ and Lmode plasmas) is found to fit both $\mathrm{H}$ - and L-mode attached plasma well, which can be interpreted as a relation with separatrix temperature $T_{e, u}, \lambda_{T_{e}, u} \propto C_{H L} q_{95} T_{e, u}^{-1}$. The dependence on $T_{e, u}$ explains why, for the same global plasma parameters, the SOL width in L-mode plasma is roughly twice in H-mode plasma. With the assumption of the existence of a different scaling for H- and L-mode plasma in SOL region, i.e. different coefficients for different regimes, a different dependence is found: $\lambda_{T_{e}, u} \propto$ $C_{H, L} q_{95}^{0.75} P_{S O L}^{-0.15}\left(C_{H}\right.$ for H-mode plasma and $C_{L}$ for L-mode plasma), which can be interpreted with a weaker dependence on separatrix temperature $T_{e, u}, \lambda_{T_{e}, u} \propto C_{H, L} q_{95} T_{e, u}^{-0.5}$.

To study the properties of plasmas with partially and completely detached divertors, a density scan at fixed plasma current and magnetic field was included in the dataset. Under detached conditions, the SOL upstream electron profile broadens when the density reaches a critical value in both $\mathrm{H}$-mode and L-mode plasma. The onset of the broadening is not consistent with the onset of divertor detachment, but consistent with the large drop of the power entering the SOL, $P_{\text {sol }}$. In fact, under H-mode completely detached condition, when $P_{\text {sol }}$ drops to the level similar as that in L-mode attached plasma, the temperature decay widths, $\lambda_{T_{e, u}}$, increase to similar values as that in L-mode attached plasma. By assuming that the parallel heat transport in the near SOL region is still dominated by conduction, upstream temperature is estimated by the same equation used in the attached regime. The widening of the SOL coincides with the dropping of upstream temperature. Combining with the data under attached and detached divertor condition, $\lambda_{T_{e}, u} \propto q_{95} T_{e, u}^{-1}$ can fit the majority of all data, except those discharges in L-mode detached regime with broader profiles, which could be due to the change of cross-field transport under detached condition being significant in L-mode

A comparison of the two scalings for $\mathrm{H}-$ and L-mode plasmas indicates that both represent the data well. The scaling with separate H-mode and L-mode coefficients has a better quality of fit, but this largely reflects the fact that this scaling has an additional fit parameter. Usually, in L-mode plasmas, the range of $P_{S O L}$ is too small to observe a change in decay width between two matched plasmas. In Hmode plasma, the present diagnostic accuracy is insufficient to detect the small change in a very thin SOL. It is believed that this is the reason why there was no observation of variation of decay width with $P_{S O L}$ in previous studies. However, within the present dataset, in L-mode plasmas with low current $(600 \mathrm{kA})$, when the SOL is much thicker, the evolution of profiles in the discharges with different $P_{S O L}$ and $q_{95}$ is more consistent with $\lambda_{T_{e}, u} \propto C_{H L} q_{95}^{0.64} P_{S O L}^{-0.32}$, i.e. $\lambda_{T_{e, u}} \propto C_{H L} q_{95} T_{e, u}^{-1}$. The study under detached divertor condition shows that the scaling, $\lambda_{T_{e}, u} \propto C_{H L} q_{95} T_{e, u}^{-1}$, describes the majority of the combined attached and detached plasmas well.

A simple relation based on power balance and Spitzer-Harm conduction is used to derive the perpendicular heat transport coefficient, $\chi_{\perp}$. The single scaling for both $\mathrm{H}$ and L-mode, implies a 
common form of perpendicular heat transport coefficient, $\chi_{\perp} \propto C_{H L}^{\chi} T_{e}^{1 / 2} / n_{e}\left(C_{H L}^{\chi}\right.$ for both H- and Lmode plasma). When the plasma becomes detached, the perpendicular transport would still be largely consistent with this dependency. The exception of L-mode detached plasma could be explained by additional physics enhancing the perpendicular transport. Using different coefficients for $\mathrm{H}$ and $\mathrm{L}$ mode plasmas, gives $\chi_{\perp} \propto C_{H, L}^{\chi} T_{e}^{3 / 2} / n_{e}\left(C_{H}^{\chi}\right.$ for H-mode plasma and $C_{L}^{\chi}$ for L-mode plasma). For this model, when the plasma becomes detached, the perpendicular transport is no longer consistent with the scaling. The present experimental results on ASDEX Upgrade have more evidence to support a universal form, $\chi_{\perp} \propto C_{H L}^{\chi} T_{e}^{1 / 2} / n_{e}$ for both $\mathrm{H}$ - and L-mode plasmas, despite of the divertor conditions. The geometric radius of the plasma, $\mathrm{R}$, and $B_{T}$ do not vary greatly within the ASDEX Upgrade database, so the dependence of the radial transport on these cannot be assessed. The scaling, $\chi_{\perp} \propto$ $C_{H L}^{\chi} T_{e}^{1 / 2} / n_{e}$, is consistent with earlier studies [21] [22] [23] [24] which used a number of theoretical models to explain the cross-field transport in the SOL. Previous results [22] of JET, taken from direct measurements using fast reciprocating probes, give an approximate correlation, $\chi_{\perp}^{S O L} \propto n_{e, u}^{-1}$. The studies [23] comparing theoretical models for the cross-field thermal diffusivities with SOL data from Compass-D, JET and Alcator C-Mod, found that the theories with a $\chi_{\perp} \propto T_{e}^{1 / 2} / n_{e}$ scaling best described the experimental data..

So, based on the dataset of this paper, there is no significant evidence for a separate H-mode and Lmode scaling and, indeed, some evidence to support a common scaling. However, the possibility of a separate scaling cannot be excluded and further experiments are required to give a definitive answer.

\section{Acknowledgements}

This work has been carried out within the framework of the EUROfusion Consortium and has received funding from the European Union's Horizon 2020 research and innovation programme under grant agreement number 633053. The views and opinions expressed herein do not necessarily reflect those of the European Commission.

\section{References}

[1] C. Pitcher and P. C. Stangeby, Plasma Phys. Control. Fusion, vol. 39, p. 779, 1997.

[2] P.C.Stangeby, The Plasma Boundary of Magnetic fusion devices, london: Institute of Physics Publishing, 2000.

[3] T. Eich et al, Phys. Rev. Lett., vol. 107, p. 215001, 2011.

[4] T. Eich et al, J. Nucl. Mater., vol. 438, p. S72, 2013.

[5] M. Makowski et al, Phys. Plasmas, vol. 19, p. 056122, 2012.

[6] T. Gray et al, J. Nucl. Mater., vol. 415, p. S360, 2011.

[7] A. J. Thornton et al, J. Nucl. Mater., vol. 438, p. S360, 2011. 
[8] B. La Bombard, Phys. Plasma, vol. 18, p. 056104, 2011.

[9] A. Scarabosio et al, J.Nucl.Mater., vol. 438, p. S426, 2013.

[10] B. Sieglin, Plasma Phys. Control. Fusion, vol. 58, p. 055015, 2016.

[11] M. Faitsch et al, Plasma Phys.Control.Fusion, vol. 57, p. 075005, 2015.

[12] H. J. Sun et al, Plasma Phys. Contr. Fusion, vol. 57, p. 125011, 2015.

[13] B. Kurzan, Rev.Sci.Instrum, vol. 74, p. 4310, 2011.

[14] S. Potzel et al, Nucl.Fusion, p. 013001, 2014.

[15] A. Kallenbach et al, Plasma Phys. Control. Fusion, vol. 46, p. 431, 2004.

[16] M. Kocan and J. P. Gunn, Plasma Phys. Controlled Fusion, vol. 52, p. 045010, 2010.

[17] D. L. Rudakov et al, J. Nucl. Materials, vol. 415, p. S387, 2011.

[18] J. Neuhauser, Plasma Phys. Control. Fusion, vol. 44, p. 855, 2002.

[19] J. Horacek et al, Plasma phys. Control Fusion, vol. 58, p. 074005, 2016.

[20] I. Paradela Pérez et al, in 22nd International Conference on Plasma Surface Interactions in Controlled Fusion Devices, Rome, Italy, 2016.

[21] S. Erents et al, Nucl.Fusion, vol. 40, p. 295, 2000.

[22] S. Erents et al, Nucl. Fusion, vol. 40, p. 309, 2000.

[23] G. Counsell, Journal of nucl.materials, Vols. 266-269, p. 91, 1999.

[24] J.W.Connor, Nucl.Fusion, vol. 39, p. 169, 1999.

[25] D.Carralero, Nucl. Fusion, vol. 54, p. 123005, 2014.

[26] D.Carralero, Physic.Review.Letter, vol. 2, p. 21500, 2015.

[27] F. Nespoli and e. al, "Understanding and suppressing the near Scrape-off layer in inboard-limited plasmas in TCV," Nucl.Fusion (submitted), 2017. 